\title{
Various spectra and energies of commuting graphs of finite rings
}

\author{
Walaa Nabil Taha Fasfous ${ }^{1}$ (D), Rajat Kanti Nath*1 (D), Reza Sharafdini ${ }^{2}$ (D) \\ ${ }^{1}$ Department of Mathematical Sciences, Tezpur University, Napaam-784028, Sonitpur, Assam, India \\ ${ }^{2}$ Department of Mathematics, Faculty of Science, Persian Gulf University, Bushehr 75169-13817, Iran
}

\begin{abstract}
The commuting graph of a non-commutative ring $R$ with center $Z(R)$ is a simple undirected graph whose vertex set is $R \backslash Z(R)$ and two vertices $x, y$ are adjacent if and only if $x y=y x$. In this paper, we compute various spectra and energies of commuting graphs of some classes of finite rings and study their consequences.
\end{abstract}

Mathematics Subject Classification (2010). 16P10, 05C50, 15A18, 05C25

Keywords. commuting graph, spectrum, energy, finite ring

\section{Introduction}

Let $R$ be a non-commutative ring with center $Z(R)$. The commuting graph of $R$, denoted by $\Gamma_{R}$, is a simple undirected graph whose vertex set is $R \backslash Z(R)$ and two vertices $x, y$ are adjacent if and only if $x y=y x$. In recent years, many mathematicians have considered commuting graph of different rings and studied various graph theoretic aspects (see $[1,3,12,13,17,19,20,23])$. Some generalizations of $\Gamma_{R}$ are also considered in $[2,9]$.

In Section 2, we compute spectrum, Laplacian spectrum and Signless Laplacian spectrum of commuting graphs of some classes of finite rings. Recall that the spectrum of a graph $\mathcal{G}$ denoted by $\operatorname{Spec}(\mathcal{G})$ is the set

$$
\left\{\lambda_{1}^{k_{1}}, \lambda_{2}^{k_{2}}, \ldots, \lambda_{n}^{k_{n}}\right\}
$$

where $\lambda_{1}, \lambda_{2}, \ldots, \lambda_{n}$ are the eigenvalues of the adjacency matrix of $\mathcal{G}$ with multiplicities $k_{1}, k_{2}, \ldots, k_{n}$ respectively. Let $A(\mathcal{G})$ and $D(\mathcal{G})$ denote the adjacency matrix and degree matrix of a graph $\mathcal{G}$ respectively. Then the Laplacian matrix and Signless Laplacian matrix of $\mathcal{G}$ are given by $L(\mathcal{G})=D(\mathcal{G})-A(\mathcal{G})$ and $Q(\mathcal{G})=D(\mathcal{G})+A(\mathcal{G})$ respectively. Let L-spec $(\mathcal{G})$ and Q-spec $(\mathcal{G})$ be the Laplacian spectrum and Signless Laplacian spectrum of $\mathcal{G}$ respectively. Then L-spec $(\mathcal{G})=\left\{\beta_{1}^{b_{1}}, \beta_{2}^{b_{2}}, \ldots, \beta_{m}^{b_{m}}\right\}$ and Q-spec $(\mathcal{G})=\left\{\gamma_{1}^{c_{1}}, \gamma_{2}^{c_{2}}, \ldots, \gamma_{n}^{c_{n}}\right\}$, where $\beta_{1}, \beta_{2}, \ldots, \beta_{m}$ are the eigenvalues of $L(\mathcal{G})$ with multiplicities $b_{1}, b_{2}, \ldots, b_{m}$ and $\gamma_{1}, \gamma_{2}, \ldots, \gamma_{n}$ are the eigenvalues of $Q(\mathcal{G})$ with multiplicities $c_{1}, c_{2}, \ldots, c_{n}$ respectively. The energy, Laplacian energy and Signless Laplacian energy of a graph $\mathcal{G}$ are given by

\footnotetext{
*Corresponding Author.

Email addresses: w.n.fasfous@gmail.com (W.N.T. Fasfous), rajatkantinath@yahoo.com (R.K. Nath), sharafdini@pgu.ac.ir (R. Sharafdini)

Received: 15.03.2019; Accepted: 24.02.2020
} 


$$
\begin{aligned}
E(\mathcal{G}) & =\sum_{\lambda \in \operatorname{Spec}(\mathcal{G})}|\lambda|, \\
L E(\mathcal{G}) & =\sum_{\mu \in \mathrm{L}-\operatorname{spec}(\mathcal{G})} \mid \mu-\frac{2|e(\mathcal{G})|}{|v(\mathcal{G})|} \text { and } \\
L E^{+}(\mathcal{G}) & =\sum_{\nu \in \mathrm{Q}-\operatorname{spec}(\mathcal{G})} \mid \nu-\frac{2|e(\mathcal{G})|}{|v(\mathcal{G})|},
\end{aligned}
$$

where $v(\mathcal{G})$ and $e(\mathcal{G})$ are the set of vertices and edges of $\mathcal{G}$, respectively.

Throughout the paper $R$ denotes a non-commutative finite ring and $p, q$ denote distinct primes. $\frac{R}{Z(R)}$ denotes the additive quotient group. Also, $K_{n}$ denotes a complete graph on $n$ vertices and $l K_{n}$ denotes the disjoint union of $l$ copies of $K_{n}$.

\section{Various spectra}

In [19], various spectra of commuting graphs of some small order finite non-commutative rings have been computed. In this section we consider more classes of finite non-commutative rings. The following theorem is useful in computing various spectra of commuting graphs of finite rings.

Theorem 2.1 ([18, Theorem 2.1]). If $\mathcal{G}=l_{1} K_{n_{1}} \sqcup l_{2} K_{n_{2}} \sqcup \cdots \sqcup l_{m} K_{n_{m}}$, then

(a) $\operatorname{Spec}(\mathcal{G})=\left\{(-1)^{\sum_{i=1}^{m} l_{i}\left(n_{i}-1\right)},\left(n_{1}-1\right)^{l_{1}},\left(n_{2}-1\right)^{l_{2}}, \ldots,\left(n_{m}-1\right)^{l_{m}}\right\}$.

(b) L-spec(G) $=\left\{0^{\sum_{i=1}^{m} l_{i}}, n_{1}^{l_{1}\left(n_{1}-1\right)}, n_{2}^{l_{2}\left(n_{2}-1\right)}, n_{m}^{l_{m}\left(n_{m}-1\right)}\right\}$.

(c) Q-spec $(\mathcal{G})=\left\{\left(2 n_{1}-2\right)^{l_{1}},\left(n_{1}-2\right)^{l_{1}\left(n_{1}-1\right)},\left(2 n_{2}-2\right)^{l_{2}},\left(n_{2}-2\right)^{l_{2}\left(n_{2}-1\right)}, \ldots\right.$,

$$
\left.\left(2 n_{m}-2\right)^{l_{m}},\left(n_{m}-2\right)^{l_{m}\left(n_{m}-1\right)}\right\} \text {. }
$$

Theorem 2.2. Let $|R|=p^{4}$ and $R$ has unity.

(a) If $|Z(R)|=p$ then $\operatorname{Spec}\left(\Gamma_{R}\right)=\left\{(-1)^{\left(p^{2}+p+1\right)\left(p^{2}-p-1\right)},\left(p^{2}-p-1\right)^{p^{2}+p+1}\right\}$, L-spec $\left(\Gamma_{R}\right)=\left\{0^{p^{2}+p+1},\left(p^{2}-p\right)^{\left(p^{2}+p+1\right)\left(p^{2}-p-1\right)}\right\} \quad$ and Q-spec $\left(\Gamma_{R}\right)=\left\{\left(2 p^{2}-2 p-2\right)^{p^{2}+p+1},\left(p^{2}-p-2\right)^{\left(p^{2}+p+1\right)\left(p^{2}-p-1\right)}\right\} ; \quad$ or $\operatorname{Spec}\left(\Gamma_{R}\right)=\left\{(-1)^{l_{1}\left(p^{2}-p-1\right)+l_{2}\left(p^{3}-p-1\right)},\left(p^{2}-p-1\right)^{l_{1}},\left(p^{3}-p-1\right)^{l_{2}}\right\}$, $\mathrm{L}-\operatorname{spec}\left(\Gamma_{R}\right)=\left\{0^{l_{1}+l_{2}},\left(p^{2}-p\right)^{l_{1}\left(p^{2}-p-1\right)},\left(p^{3}-p\right)^{l_{2}\left(p^{3}-p-1\right)}\right\}$ and Q-spec $\left(\Gamma_{R}\right)=$ $\left\{\left(2 p^{2}-2 p-2\right)^{l_{1}},\left(p^{2}-p-2\right)^{l_{1}\left(p^{2}-p-1\right)},\left(2 p^{3}-2 p-2\right)^{l_{2}},\left(p^{3}-p-2\right)^{l_{2}\left(p^{3}-p-1\right)}\right\}$, where $l_{1}+l_{2}(p+1)=p^{2}+p+1$.

(b) If $|Z(R)|=p^{2}$ then $\operatorname{Spec}\left(\Gamma_{R}\right)=\left\{(-1)^{(p+1)\left(p^{3}-p^{2}-1\right)},\left(p^{3}-p^{2}-1\right)^{p+1}\right\}$,

L-spec $\left(\Gamma_{R}\right)=\left\{0^{p+1},\left(p^{3}-p^{2}\right)^{(p+1)\left(p^{3}-p^{2}-1\right)}\right\}$ and Q-spec $\left(\Gamma_{R}\right)=\left\{\left(2 p^{3}-2 p^{2}-2\right)^{p+1},\left(p^{3}-p^{2}-2\right)^{(p+1)\left(p^{3}-p^{2}-1\right)}\right\}$.

Proof. (a) If $|Z(R)|=p$ then, by Theorem 2.5 of [22], we have $\Gamma_{R}=\left(p^{2}+p+1\right) K_{\left(p^{2}-p\right)}$ or $l_{1} K_{\left(p^{2}-p\right)} \sqcup l_{2} K_{\left(p^{3}-p\right)}$, where $l_{1}+l_{2}(p+1)=p^{2}+p+1$. Hence, the result follows from Theorem 2.1.

(b) If $|Z(R)|=p^{2}$ then, by Theorem 2.5 of [22], we have $\Gamma_{R}=(p+1) K_{\left(p^{3}-p^{2}\right)}$. Hence, the result follows from Theorem 2.1. 
Theorem 2.3. Let $|R|=p^{5}$ with unity and $Z(R)$ is not a field.

(a) If $|Z(R)|=p^{2}$ then $\operatorname{Spec}\left(\Gamma_{R}\right)=\left\{(-1)^{\left(p^{2}+p+1\right)\left(p^{3}-p^{2}-1\right)},\left(p^{3}-p^{2}-1\right)^{p^{2}+p+1}\right\}$,

L-spec $\left(\Gamma_{R}\right)=\left\{0^{p^{2}+p+1},\left(p^{3}-p^{2}\right)^{\left(p^{2}+p+1\right)\left(p^{3}-p^{2}-1\right)}\right\} \quad$ and

Q-spec $\left(\Gamma_{R}\right)=\left\{\left(2 p^{3}-2 p^{2}-2\right)^{p^{2}+p+1},\left(p^{3}-p^{2}-2\right)^{\left(p^{2}+p+1\right)\left(p^{3}-p^{2}-1\right)}\right\} ; \quad$ or

$\operatorname{Spec}\left(\Gamma_{R}\right)=\left\{(-1)^{l_{1}\left(p^{3}-p^{2}-1\right)+l_{2}\left(p^{3}-p-1\right)},\left(p^{3}-p^{2}-1\right)^{l_{1}},\left(p^{3}-p-1\right)^{l_{2}}\right\}$,

L-spec $\left(\Gamma_{R}\right)=\left\{0^{l_{1}+l_{2}},\left(p^{3}-p^{2}\right)^{l_{1}\left(p^{3}-p^{2}-1\right)},\left(p^{3}-p\right)^{l_{2}\left(p^{3}-p-1\right)}\right\} \quad$ and

Q-spec $\left(\Gamma_{R}\right)=\left\{\left(2 p^{3}-2 p^{2}-2\right)^{l_{1}},\left(p^{3}-p^{2}-2\right)^{l_{1}\left(p^{3}-p^{2}-1\right)},\left(2 p^{3}-2 p-2\right)^{l_{2}}\right.$,

$\left.\left(p^{3}-p-2\right)^{l_{2}\left(p^{3}-p-1\right)}\right\}$, where $l_{1}+l_{2}(p+1)=p^{2}+p+1$.

(b) If $|Z(R)|=p^{3}$ then $\operatorname{Spec}\left(\Gamma_{R}\right)=\left\{(-1)^{(p+1)\left(p^{4}-p^{3}-1\right)},\left(p^{4}-p^{3}-1\right)^{p+1}\right\}$,

$\mathrm{L}-\operatorname{spec}\left(\Gamma_{R}\right)=\left\{0^{p+1},\left(p^{4}-p^{3}\right)^{(p+1)\left(p^{4}-p^{3}-1\right)}\right\} \quad$ and

Q-spec $\left(\Gamma_{R}\right)=\left\{\left(2 p^{4}-2 p^{3}-2\right)^{p+1},\left(p^{4}-p^{3}-2\right)^{(p+1)\left(p^{4}-p^{3}-1\right)}\right\}$.

Proof. (a) If $|Z(R)|=p^{2}$ then, by Theorem 2.7 of [22], we have $\Gamma_{R}=\left(p^{2}+p+1\right) K_{\left(p^{3}-p^{2}\right)}$ or $l_{1} K_{\left(p^{3}-p^{2}\right)} \sqcup l_{2} K_{\left(p^{3}-p\right)}$, where $l_{1}+l_{2}(p+1)=p^{2}+p+1$. Hence, the result follows from Theorem 2.1.

(b) If $|Z(R)|=p^{3}$ then, by Theorem 2.7 of [22], we have $\Gamma_{R}=(p+1) K_{\left(p^{4}-p^{3}\right)}$. Hence, the result follows from Theorem 2.1.

Theorem 2.4. Let $|R|=p q$ and $Z(R)=\{0\}$.

(a) If $(p-1) \mid(p q-1)$ then $\operatorname{Spec}\left(\Gamma_{R}\right)=\left\{(-1)^{\frac{(p q-1)(p-2)}{p-1}},(p-2)^{\frac{p q-1}{p-1}}\right\}$, L-spec $\left(\Gamma_{R}\right)=$ $\left\{0^{\frac{p q-1}{p-1}},(p-1)^{\frac{(p q-1)(p-2)}{p-1}}\right\}$ and $\mathrm{Q}-\operatorname{spec}\left(\Gamma_{R}\right)=\left\{(2 p-4)^{\frac{p q-1}{p-1}},(p-3)^{\frac{(p q-1)(p-2)}{p-1}}\right\}$.

(b) If $(q-1) \mid(p q-1)$ then $\operatorname{Spec}\left(\Gamma_{R}\right)=\left\{(-1)^{\frac{(p q-1)(q-2)}{q-1}},(q-2)^{\frac{p q-1}{q-1}}\right\}$, L-spec $\left(\Gamma_{R}\right)=$ $\left\{0^{\frac{p q-1}{q-1}},(q-1)^{\frac{(p q-1)(q-2)}{q-1}}\right\}$ and $\mathrm{Q}-\operatorname{spec}\left(\Gamma_{R}\right)=\left\{(2 q-4)^{\frac{p q-1}{q-1}},(q-3)^{\frac{(p q-1)(q-2)}{q-1}}\right\}$.

(c) If $l_{1}(p-1)+l_{2}(q-1)=p q-1$ then $\operatorname{Spec}\left(\Gamma_{R}\right)=\left\{(-1)^{l_{1}(p-2)+l_{2}(q-2)},(p-2)^{l_{1}}\right.$, $\left.(q-2)^{l_{2}}\right\}$, L-spec $\left(\Gamma_{R}\right)=\left\{0^{l_{1}+l_{2}},(p-1)^{l_{1}(p-2)},(q-1)^{l_{2}(q-2)}\right\} \quad$ and $\mathrm{Q}-\operatorname{spec}\left(\Gamma_{R}\right)=\left\{(2 p-4)^{l_{1}},(p-3)^{l_{1}(p-2)},(2 q-4)^{l_{2}},(q-3)^{l_{2}(q-2)}\right\}$.

Proof. It was shown in [23, Theorem 2.8] that

$$
\Gamma_{R}= \begin{cases}\frac{p q-1}{p-1} K_{p-1}, & \text { if }(p-1) \mid(p q-1) \\ \frac{p q-1}{q-1} K_{q-1}, & \text { if }(q-1) \mid(p q-1) \\ l_{1} K_{p-1} \sqcup l_{2} K_{q-1}, & \text { if } l_{1}(p-1)+l_{2}(q-1)=p q-1 .\end{cases}
$$

Hence, the result follows from Theorem 2.1.

Theorem 2.5. Let $|R|=p^{2} q$ and $Z(R)=\{0\}$.

(a) If $t \in\left\{p, q, p^{2}, p q\right\}$ and $(t-1) \mid\left(p^{2} q-1\right)$ then $\operatorname{Spec}\left(\Gamma_{R}\right)=\left\{(-1)^{\frac{\left(p^{2} q-1\right)(t-2)}{t-1}}\right.$,

$$
\left.(t-2)^{\frac{p^{2} q-1}{t-1}}\right\}, \quad \operatorname{L-spec}\left(\Gamma_{R}\right)=\left\{0^{\frac{p^{2} q-1}{t-1}},(t-1)^{\frac{\left(p^{2} q-1\right)(t-2)}{t-1}}\right\} \quad \text { and }
$$

Q-spec $\left(\Gamma_{R}\right)=\left\{(2 t-4)^{\frac{p^{2} q-1}{t-1}},(t-3)^{\frac{\left(p^{2} q-1\right)(t-2)}{t-1}}\right\}$.

(b) If $l_{1}(p-1)+l_{2}(q-1)+l_{3}\left(p^{2}-1\right)+l_{4}(p q-1)=p^{2} q-1$ then $\operatorname{Spec}\left(\Gamma_{R}\right)$ $=\left\{(-1)^{l_{1}(p-2)+l_{2}(q-2)+l_{3}\left(p^{2}-2\right)+l_{4}(p q-2)},(p-2)^{l_{1}},(q-2)^{l_{2}},\left(p^{2}-2\right)^{l_{3}},(p q-2)^{l_{4}}\right\}$, 


$$
\begin{aligned}
& \text { L-spec }\left(\Gamma_{R}\right)=\left\{0^{l_{1}+l_{2}+l_{3}+l_{4}},(p-1)^{l_{1}(p-2)},(q-1)^{l_{2}(q-2)},\left(p^{2}-1\right)^{l_{3}\left(p^{2}-2\right)},\right. \\
& \left.(p q-1)^{l_{4}(p q-2)}\right\} \quad \text { and } \quad \mathrm{Q}-\operatorname{spec}\left(\Gamma_{R}\right)=\left\{(2 p-4)^{l_{1}},(p-3)^{l_{1}(p-2)},(2 q-4)^{l_{2}},\right. \\
& \left.(q-3)^{l_{2}(q-2)},\left(2 p^{2}-4\right)^{l_{3}},\left(p^{2}-3\right)^{l_{3}\left(p^{2}-2\right)},(2 p q-4)^{l_{4}},(p q-3)^{l_{4}(p q-2)}\right\} .
\end{aligned}
$$

Proof. (a) By [23, Theorem 2.9], we have $\Gamma_{R}=\frac{p^{2} q-1}{t-1} K_{t-1}$ if $t \in\left\{p, q, p^{2}, p q\right\}$ and $(t-1) \mid$ $\left(p^{2} q-1\right)$. Hence, the result follows from Theorem 2.1 .

(b) By [23, Theorem 2.9], we also have $\Gamma_{R}=l_{1} K_{p-1} \sqcup l_{2} K_{q-1} \sqcup l_{3} K_{p^{2}-1} \sqcup l_{4} K_{p q-1}$ if $l_{1}(p-1)+l_{2}(q-1)+l_{3}\left(p^{2}-1\right)+l_{4}(p q-1)=p^{2} q-1$. Hence, the result follows from Theorem 2.1 .

Theorem 2.6. Let $|R|=p^{3} q$ and $R$ has unity. If $|Z(R)|=p q$ then

$$
\begin{aligned}
\operatorname{Spec}\left(\Gamma_{R}\right) & =\left\{(-1)^{(p+1)\left(p^{2} q-p q-1\right)},\left(p^{2} q-p q-1\right)^{p+1}\right\}, \\
\text { L-spec }\left(\Gamma_{R}\right) & =\left\{0^{p+1},\left(p^{2} q-p q\right)^{(p+1)\left(p^{2} q-p q-1\right)}\right\} \text { and } \\
\text { Q-spec }\left(\Gamma_{R}\right) & =\left\{\left(2 p^{2} q-2 p q-2\right)^{p+1},\left(p^{2} q-p q-2\right)^{(p+1)\left(p^{2} q-p q-1\right)}\right\} .
\end{aligned}
$$

Proof. If $|Z(R)|=p q$ then, by [23, Theorem 2.12], we have $\Gamma_{R}=(p+1) K_{p^{2} q-p q}$. Hence, the result follows from Theorem 2.1.

We conclude this section with the following result.

Theorem 2.7. Let $|R|=p^{3} q$, $R$ has unity and $|Z(R)|=p^{2}$.

(a) If $(p-1) \mid(p q-1)$ then $\operatorname{Spec}\left(\Gamma_{R}\right)=\left\{(-1)^{\frac{(p q-1)\left(p^{3}-p^{2}-1\right)}{p-1}},\left(p^{3}-p^{2}-1\right)^{\frac{p q-1}{p-1}}\right\}$,

$$
\begin{aligned}
& \text { L-spec }\left(\Gamma_{R}\right)=\left\{0^{\frac{p q-1}{p-1}},\left(p^{3}-p^{2}\right)^{\frac{(p q-1)\left(p^{3}-p^{2}-1\right)}{p-1}}\right\} \text { and } \\
& \text { Q-spec }\left(\Gamma_{R}\right)=\left\{\left(2 p^{3}-2 p^{2}-2\right)^{\frac{p q-1}{p-1}},\left(p^{3}-p^{2}-2\right)^{\frac{(p q-1)\left(p^{3}-p^{2}-1\right)}{p-1}}\right\} .
\end{aligned}
$$

(b) If $(q-1) \mid(p q-1)$ then $\operatorname{Spec}\left(\Gamma_{R}\right)=\left\{(-1)^{\frac{(p q-1)\left(p^{2} q-p^{2}-1\right)}{q-1}},\left(p^{2} q-p^{2}-1\right)^{\frac{p q-1}{q-1}}\right\}$,

$$
\begin{aligned}
& \text { L-spec }\left(\Gamma_{R}\right)=\left\{0^{\frac{p q-1}{q-1}},\left(p^{2} q-p^{2}\right)^{\left.\frac{(p q-1)\left(p^{2} q-p^{2}-1\right)}{q-1}\right\} \text { and }}\right. \\
& \text { Q-spec }\left(\Gamma_{R}\right)=\left\{\left(2 p^{2} q-2 p^{2}-2\right)^{\frac{p q-1}{q-1}},\left(p^{2} q-p^{2}-2\right)^{\frac{(p q-1)\left(p^{2} q-p^{2}-1\right)}{q-1}}\right\} .
\end{aligned}
$$

(c) If $l_{1}(p-1)+l_{2}(q-1)=p q-1$ then

$$
\begin{aligned}
& \operatorname{Spec}\left(\Gamma_{R}\right)=\{\left.(-1)^{l_{1}\left(p^{3}-p^{2}-1\right)+l_{2}\left(p^{2} q-p^{2}-1\right)},\left(p^{3}-p^{2}-1\right)^{l_{1}},\left(p^{2} q-p^{2}-1\right)^{l_{2}}\right\}, \\
& \operatorname{L}-\operatorname{spec}\left(\Gamma_{R}\right)=\left\{0^{l_{1}+l_{2}},\left(p^{3}-p^{2}\right)^{l_{1}\left(p^{3}-p^{2}-1\right)},\left(p^{2} q-p^{2}\right)^{l_{2}\left(p^{2} q-p^{2}-1\right)}\right\} \quad \text { and } \\
& \mathrm{Q}-\operatorname{spec}\left(\Gamma_{R}\right)=\left\{\left(2 p^{3}-2 p^{2}-2\right)^{l_{1}},\left(p^{3}-p^{2}-2\right)^{l_{1}\left(p^{3}-p^{2}-1\right)},\left(2 p^{2} q-2 p^{2}-2\right)^{l_{2}},\right. \\
&\left.\left(p^{2} q-p^{2}-2\right)^{l_{2}\left(p^{2} q-p^{2}-1\right)}\right\} .
\end{aligned}
$$

Proof. If $|Z(R)|=p^{2}$ then, by [23, Theorem 2.12], we have

$$
\Gamma_{R}= \begin{cases}\frac{p q-1}{p-1} K_{p^{3}-p^{2}}, & \text { if }(p-1) \mid(p q-1) \\ \frac{p q-1}{q-1} K_{p^{2} q-p^{2}}, & \text { if }(q-1) \mid(p q-1) \\ l_{1} K_{p^{3}-p^{2}} \sqcup l_{2} K_{p^{2} q-p^{2}}, & \text { if } l_{1}(p-1)+l_{2}(q-1)=p q-1 .\end{cases}
$$

Hence, the result follows from Theorem 2.1. 


\section{Various energies}

If $\frac{R}{Z(R)}$ is isomorphic to $\mathbb{Z}_{p} \times \mathbb{Z}_{p}$, then it was shown in Theorem 3.1 of [19] that

$$
E\left(\Gamma_{R}\right)=L E\left(\Gamma_{R}\right)=L E^{+}\left(\Gamma_{R}\right)=2\left(p^{2}-1\right)|Z(R)|-2(p+1) .
$$

As a consequence of (3.1), in the following results, we compute various energies of commuting graphs of several well-known classes of finite rings.

Theorem 3.1. If $|R|=p^{2}$, then $E\left(\Gamma_{R}\right)=L E\left(\Gamma_{R}\right)=L E^{+}\left(\Gamma_{R}\right)=2\left(p^{2}-p-2\right)$.

Proof. If $R$ is a non-commutative ring of order $p^{2}$, then $Z(R)$ has only one element. Therefore, the additive group $\frac{R}{Z(R)} \cong \mathbb{Z}_{p} \times \mathbb{Z}_{p}$. Hence the result follows from (3.1).

Theorem 3.2. If $|R|=p^{3}$ and $R$ has unity, then

$$
E\left(\Gamma_{R}\right)=L E\left(\Gamma_{R}\right)=L E^{+}\left(\Gamma_{R}\right)=2\left(p^{3}-2 p-1\right) .
$$

Proof. If $R$ is a non-commutative ring with unity of order $p^{3}$, then $Z(R)$ has $p$ elements. Therefore, the additive group $\frac{R}{Z(R)} \cong \mathbb{Z}_{p} \times \mathbb{Z}_{p}$. Hence the result follows from (3.1).

A ring $R$ is called an $n$-centralizer ring if $|\operatorname{Cent}(R)|=n$, where $\operatorname{Cent}(R)=\left\{C_{R}(x): x \in\right.$ $R\}$. Various properties of $n$-centralizer rings can be found in $[5,10,11]$. In the following results we compute various energies of some finite $n$-centralizer rings.

Theorem 3.3. If $|\operatorname{Cent}(R)|=4$, then $E\left(\Gamma_{R}\right)=L E\left(\Gamma_{R}\right)=L E^{+}\left(\Gamma_{R}\right)=6|Z(R)|-6$.

Proof. It was shown in [11, Theorem 3.2] that the additive quotient group $\frac{R}{Z(R)} \cong \mathbb{Z}_{2} \times \mathbb{Z}_{2}$ if $R$ is a finite 4-centralizer ring. Hence, the result follows from (3.1) putting $p=2$.

Theorem 3.4. If $|\operatorname{Cent}(R)|=5$, then $E\left(\Gamma_{R}\right)=L E\left(\Gamma_{R}\right)=L E^{+}\left(\Gamma_{R}\right)=16|Z(R)|-8$.

Proof. It was shown in [11, Theorem 4.3] that the additive quotient group $\frac{R}{Z(R)} \cong \mathbb{Z}_{3} \times \mathbb{Z}_{3}$ if $R$ is a finite 5-centralizer ring. Hence, the result follows from (3.1).

Theorem 3.5. If $R$ is a finite $p$-ring and $|\operatorname{Cent}(R)|=(p+2)$, then

$$
E\left(\Gamma_{R}\right)=L E\left(\Gamma_{R}\right)=L E^{+}\left(\Gamma_{R}\right)=2\left(p^{2}-1\right)|Z(R)|-2(p+1) .
$$

Proof. It was shown in [11, Theorem 2.12] that the additive quotient group $\frac{R}{Z(R)} \cong \mathbb{Z}_{p} \times \mathbb{Z}_{p}$ if $R$ is a finite $(p+2)$-centralizer $p$-ring. Hence, the result follows from (3.1).

In 1976, MacHale [16] initiated the study of commutativity degree of a finite ring $R$ denoted by $\operatorname{Pr}(R)$. Recall that the commutativity degree of $R$ is the probability that a randomly chosen pair of elements of $R$ commute. Recent results on $\operatorname{Pr}(R)$ can be found in [4,6-8]. In the following theorem we compute various energies of $\Gamma_{R}$ for some given values of $\operatorname{Pr}(R)$.

Theorem 3.6. Let $p$ be the smallest prime dividing $|R|$. If $\operatorname{Pr}(R)=\frac{p^{2}+p-1}{p^{3}}$ then

$$
E\left(\Gamma_{R}\right)=L E\left(\Gamma_{R}\right)=L E^{+}\left(\Gamma_{R}\right)=2\left(p^{2}-1\right)|Z(R)|-2(p+1) .
$$

Proof. By Theorem 2 of [16] we have $\frac{R}{Z(R)} \cong \mathbb{Z}_{p} \times \mathbb{Z}_{p}$. Hence, the result follows from (3.1).

We have the following corollary to the above theorem.

Corollary 3.7. If $\operatorname{Pr}(R)=\frac{5}{8}$ then

$$
E\left(\Gamma_{R}\right)=L E\left(\Gamma_{R}\right)=L E^{+}\left(\Gamma_{R}\right)=6|Z(R)|-6 .
$$

Now we compute various energies of $\Gamma_{R}$ for the rings considered in Section 2. Note that one can do this using Theorems $2.1-2.7$ and (1.1). However, using the following theorem one can also compute various energies. 
Theorem 3.8. If $\mathcal{G}=l_{1} K_{n_{1}} \sqcup l_{2} K_{n_{2}}$, then $E(\mathcal{G})=2 l_{1}\left(n_{1}-1\right)+2 l_{2}\left(n_{2}-1\right)$. Further, if $n_{1}=n_{2}=n$ then

$$
E\left(l K_{n}\right)=L E\left(l K_{n}\right)=L E^{+}\left(l K_{n}\right)=2 l(n-1),
$$

where $l=l_{1}+l_{2}$.

Proof. By Theorem 2.1(a) we have

$$
\operatorname{Spec}(\mathcal{G})=\left\{(-1)^{\sum_{i=1}^{2} l_{i}\left(n_{i}-1\right)},\left(n_{1}-1\right)^{l_{1}},\left(n_{2}-1\right)^{l_{2}}\right\} .
$$

Therefore, (1.1) gives

$$
\begin{aligned}
E(\mathcal{G}) & =|-1| \sum_{i=1}^{2} l_{i}\left(n_{i}-1\right)+l_{1}\left|n_{1}-1\right|+l_{2}\left|n_{2}-1\right| \\
& =l_{1}\left(n_{1}-1\right)+l_{2}\left(n_{2}-1\right)+l_{1}\left(n_{1}-1\right)+l_{2}\left(n_{2}-1\right) \\
& =2 l_{1}\left(n_{1}-1\right)+2 l_{2}\left(n_{2}-1\right) .
\end{aligned}
$$

If $n_{1}=n_{2}=n$ and $l=l_{1}+l_{2}$ then $\mathcal{G}=l K_{n}$ and so $E\left(l K_{n}\right)=2 l(n-1)$. In this case, we also have $\left|v\left(l K_{n}\right)\right|=\ln ,\left|e\left(l K_{n}\right)\right|=\frac{\ln (n-1)}{2}$ and so $\frac{2\left|e\left(l K_{n}\right)\right|}{\left|v\left(l K_{n}\right)\right|}=n-1$.

By Theorem 2.1(b) we have L-spec $\left(l K_{n}\right)=\left\{0^{l}, n^{l(n-1)}\right\}$. Therefore

$$
\left|0-\frac{2\left|e\left(l K_{n}\right)\right|}{\left|v\left(l K_{n}\right)\right|}\right|=n-1 \text { and }\left|n-\frac{2\left|e\left(l K_{n}\right)\right|}{\left|v\left(l K_{n}\right)\right|}\right|=1 .
$$

Hence, (1.1) gives

$$
L E\left(l K_{n}\right)=(n-1) l+l(n-1)=2 l(n-1) .
$$

Again, by Theorem 2.1(c) we also have Q-spec $\left(l K_{n}\right)=\left\{(2 n-2)^{l},(n-2)^{l(n-1)}\right\}$. Therefore

Hence, (1.1) gives

$$
\left|2 n-2-\frac{2\left|e\left(l K_{n}\right)\right|}{\left|v\left(l K_{n}\right)\right|}\right|=n-1 \text { and }\left|n-2-\frac{2\left|e\left(l K_{n}\right)\right|}{\left|v\left(l K_{n}\right)\right|}\right|=1 .
$$

$$
L E^{+}\left(l K_{n}\right)=(n-1) l+l(n-1)=2 l(n-1) .
$$

This completes the proof.

Theorem 3.9. Let $R$ have unity and $|R|=p^{4}$.

(a) If $|Z(R)|=p$ then $E\left(\Gamma_{R}\right)=L E\left(\Gamma_{R}\right)=L E^{+}\left(\Gamma_{R}\right)=2\left(p^{2}+p+1\right)\left(p^{2}-p-1\right)$ or $E\left(\Gamma_{R}\right)=2 l_{1}\left(p^{2}-p-1\right)+2 l_{2}\left(p^{3}-p-1\right)$, where $l_{1}+l_{2}(p+1)=p^{2}+p+1$.

(b) If $|Z(R)|=p^{2}$ then $E\left(\Gamma_{R}\right)=L E\left(\Gamma_{R}\right)=L E^{+}\left(\Gamma_{R}\right)=2(p+1)\left(p^{3}-p^{2}-1\right)$.

Proof. By Theorem 2.5 of [22], we have $\Gamma_{R}=\left(p^{2}+p+1\right) K_{\left(p^{2}-p\right)}$ or $l_{1} K_{\left(p^{2}-p\right)} \sqcup l_{2} K_{\left(p^{3}-p\right)}$ (where $\left.l_{1}+l_{2}(p+1)=p^{2}+p+1\right)$ if $|Z(R)|=p$ and $(p+1) K_{\left(p^{3}-p^{2}\right)}$ if $|Z(R)|=p^{2}$. Hence, the result follows from Theorem 3.8.

Theorem 3.10. Let $R$ have unity, $|R|=p^{5}$ and $Z(R)$ is not a field.

(a) If $|Z(R)|=p^{2}$ then $E\left(\Gamma_{R}\right)=L E\left(\Gamma_{R}\right)=L E^{+}\left(\Gamma_{R}\right)=2\left(p^{2}+p+1\right)\left(p^{3}-p^{2}-1\right)$ or $E\left(\Gamma_{R}\right)=2 l_{1}\left(p^{3}-p^{2}-1\right)+2 l_{2}\left(p^{3}-p-1\right)$, where $l_{1}+l_{2}(p+1)=p^{2}+p+1$.

(b) If $|Z(R)|=p^{3}$ then $E\left(\Gamma_{R}\right)=L E\left(\Gamma_{R}\right)=L E^{+}\left(\Gamma_{R}\right)=2(p+1)\left(p^{4}-p^{3}-1\right)$.

Proof. By Theorem 2.7 of [22], we have $\Gamma_{R}=\left(p^{2}+p+1\right) K_{\left(p^{3}-p^{2}\right)}$ or $l_{1} K_{\left(p^{3}-p^{2}\right)} \sqcup l_{2} K_{\left(p^{3}-p\right)}$ (where $\left.l_{1}+l_{2}(p+1)=p^{2}+p+1\right)$ if $|Z(R)|=p^{2}$ and $(p+1) K_{\left(p^{4}-p^{3}\right)}$ if $|Z(R)|=p^{3}$. Hence, the result follows from Theorem 3.8. 
Theorem 3.11. Let $|R|=p q$ and $Z(R)=\{0\}$.

(a) If $(p-1) \mid(p q-1)$ then $E\left(\Gamma_{R}\right)=L E\left(\Gamma_{R}\right)=L E^{+}\left(\Gamma_{R}\right)=\frac{2(p q-1)(p-2)}{p-1}$.

(b) If $(q-1) \mid(p q-1)$ then $E\left(\Gamma_{R}\right)=L E\left(\Gamma_{R}\right)=L E^{+}\left(\Gamma_{R}\right)=\frac{2(p q-1)(q-2)}{q-1}$.

(c) If $l_{1}(p-1)+l_{2}(q-1)=p q-1$ then $E\left(\Gamma_{R}\right)=2 l_{1}(p-2)+2 l_{2}(q-2)$.

Proof. It was shown in [23, Theorem 2.8] that

$$
\Gamma_{R}= \begin{cases}\frac{p q-1}{p-1} K_{p-1}, & \text { if }(p-1) \mid(p q-1) \\ \frac{p q-1}{q-1} K_{q-1}, & \text { if }(q-1) \mid(p q-1) \\ l_{1} K_{p-1} \sqcup l_{2} K_{q-1}, & \text { if } l_{1}(p-1)+l_{2}(q-1)=p q-1 .\end{cases}
$$

Hence, the result follows from Theorem 3.8.

Theorem 3.12. Let $|R|=p^{2} q$ and $Z(R)=\{0\}$.

(a) If $t \in\left\{p, q, p^{2}, p q\right\}$ and $(t-1) \mid\left(p^{2} q-1\right)$ then

$$
E\left(\Gamma_{R}\right)=L E\left(\Gamma_{R}\right)=L E^{+}\left(\Gamma_{R}\right)=\frac{2\left(p^{2} q-1\right)(t-2)}{t-1} .
$$

(b) If $l_{1}(p-1)+l_{2}(q-1)+l_{3}\left(p^{2}-1\right)+l_{4}(p q-1)=p^{2} q-1$ then

$$
E\left(\Gamma_{R}\right)=2\left(p^{2} q-1-\left(l_{1}+l_{2}+l_{3}+l_{4}\right) .\right.
$$

Proof. (a) By [23, Theorem 2.9], we have $\Gamma_{R}=\frac{p^{2} q-1}{t-1} K_{t-1}$ if $t \in\left\{p, q, p^{2}, p q\right\}$ and $(t-1) \mid$ $\left(p^{2} q-1\right)$. Hence, the result follows from Theorem 3.8 .

(b) By [23, Theorem 2.9], we also have $\Gamma_{R}=l_{1} K_{p-1} \sqcup l_{2} K_{q-1} \sqcup l_{3} K_{p^{2}-1} \sqcup l_{4} K_{p q-1}$ if $l_{1}(p-1)+l_{2}(q-1)+l_{3}\left(p^{2}-1\right)+l_{4}(p q-1)=p^{2} q-1$. Hence, the result follows from Theorem 2.5 and (1.1).

Theorem 3.13. Let $R$ have unity and $|R|=p^{3} q$. If $|Z(R)|=p q$ then

$$
E\left(\Gamma_{R}\right)=L E\left(\Gamma_{R}\right)=L E^{+}\left(\Gamma_{R}\right)=2(p+1)\left(p^{2} q-p q-1\right) .
$$

Proof. If $|Z(R)|=p q$ then, by [23, Theorem 2.12], we have $\Gamma_{R}=(p+1) K_{p^{2} q-p q}$. Hence the result follows from Theorem 3.8.

Theorem 3.14. Let $R$ have unity, $|R|=p^{3} q$ and $|Z(R)|=p^{2}$.

(a) If $(p-1) \mid(p q-1)$ then $E\left(\Gamma_{R}\right)=L E\left(\Gamma_{R}\right)=L E^{+}\left(\Gamma_{R}\right)=\frac{2(p q-1)\left(p^{3}-p^{2}-1\right)}{p-1}$.

(b) If $(q-1) \mid(p q-1)$ then

$$
E\left(\Gamma_{R}\right)=L E\left(\Gamma_{R}\right)=L E^{+}\left(\Gamma_{R}\right)=\frac{2(p q-1)\left(p^{2} q-p^{2}-1\right)}{q-1} .
$$

(c) If $l_{1}(p-1)+l_{2}(q-1)=p q-1$ then

$$
E\left(\Gamma_{R}\right)=2 l_{1}\left(p^{3}-p^{2}-1\right)+2 l_{2}\left(p^{2} q-p^{2}-1\right) .
$$

Proof. If $|Z(R)|=p^{2}$ then, by [23, Theorem 2.12], we have

$$
\Gamma_{R}= \begin{cases}\frac{p q-1}{p-1} K_{p^{3}-p^{2}}, & \text { if }(p-1) \mid(p q-1) \\ \frac{p q-1}{q-1} K_{p^{2} q-p^{2}}, & \text { if }(q-1) \mid(p q-1) \\ l_{1} K_{p^{3}-p^{2}} \sqcup l_{2} K_{p^{2} q-p^{2}}, & \text { if } l_{1}(p-1)+l_{2}(q-1)=p q-1 .\end{cases}
$$

Hence, the result follows from Theorem 3.8.

Note that the rings considered above are CC-rings. Recall that a non-commutative ring $R$ is called a CC-ring if all the centralizers of its non-central elements are commutative. In other words, $C_{R}(x)$ for all $x \in R \backslash Z(R)$ is commutative, where $C_{R}(x):=\{y \in R: x y=y x\}$ is the centralizer of $x$. The study of CC-rings was initiated by Erfanian et al. in [13]. In the following theorem we compute energy of a CC-ring. 
Theorem 3.15. Let $R$ be a finite CC-ring with distinct centralizers $S_{1}, S_{2}, \ldots, S_{n}$ of non-central elements of $R$. Then $E\left(\Gamma_{R}\right)=2(|R|-|Z(R)|-n)$.

Proof. By Theorem 2.1 of [12] we have

$$
\operatorname{Spec}\left(\Gamma_{R}\right)=\left\{(-1)^{\sum_{i=1}^{n}\left|S_{i}\right|-n(|Z(R)|+1)},\left(\left|S_{1}\right|-|Z(R)|-1\right)^{1}, \ldots,\left(\left|S_{n}\right|-|Z(R)|-1\right)^{1}\right\} .
$$

Therefore

$$
\begin{array}{rlr}
E\left(\Gamma_{R}\right) & =\sum_{i=1}^{n}\left|S_{i}\right|-n(|Z(R)|+1)+\left(\left|S_{1}\right|-|Z(R)|-1\right)+\cdots \\
& +\left(\left|S_{n}\right|-|Z(R)|-1\right) \\
& =2 \sum_{i=1}^{n}\left|S_{i}\right|-2 n|Z(R)|-2 n .
\end{array}
$$

Since $\sum_{i=1}^{n}\left|S_{i}\right|=|R|+(n-1)|Z(R)|$, we get the required expression for $E\left(\Gamma_{R}\right)$.

Corollary 3.16. Let $R$ be a finite $C C$-ring and $A$ be any finite commutative ring. Then $E\left(\Gamma_{R \times A}\right)=2(|R||A|-|Z(R)||A|-n)$, where $n=|\operatorname{Cent}(R)|-1$.

Proof. Follows from Theorem 3.15 noting that $R \times A$ is a CC-ring, $|\operatorname{Cent}(R)|=\mid \operatorname{Cent}(R \times$ $A) \mid$ and $Z(R \times A)=Z(R) \times A$.

\section{Some consequences}

A finite non-commutative ring $R$ is called super integral if spectrum, Laplacian spectrum and Signless Laplacian spectrum of $\Gamma_{R}$ contain only integers. The notion of super integral ring was introduced in [19]. It can be seen that all the rings considered in Section 2 are super integral.

A finite graph $\mathcal{G}$ is called hyperenergetic and borderenergetic if $E(\mathcal{G})>E\left(K_{|v(\mathcal{G})|}\right)$ and $E(\mathcal{G})=E\left(K_{|v(\mathcal{G})|}\right)$ respectively. Similarly, $\mathcal{G}$ is called L-hyperenergetic and L-borderenergetic if $L E(\mathcal{G})>L E\left(K_{|v(\mathcal{G})|}\right)$ and $L E(\mathcal{G})=L E\left(K_{|v(\mathcal{G})|}\right)$ respectively; $\mathcal{G}$ is called Q-hyperenergetic and Q-borderenergetic if $L E^{+}(\mathcal{G})>L E^{+}\left(K_{|v(\mathcal{G})|}\right)$ and $L E^{+}(\mathcal{G})=$ $L E^{+}\left(K_{|v(\mathcal{G})|}\right)$ respectively. The study of hyperenergetic graph was initiated by Walikar et al. [24] and Gutman [15] in 1999. The concepts of borderenergetic and L-borderenergetic graphs were introduced by Gong et al. [14] and Tura [21] in the years 2015 and 2017 respectively.

A finite graph $\mathcal{G}$ is called super hyperenergetic if it is hyperenergetic, L-hyperenergetic and Q-hyperenergetic. Similarly, we define super borderenergetic graph. In this section, we show that the commuting graphs of the rings considered in Section 3 are neither super hyperenergetic nor super borderenergetic.

Theorem 4.1. If $\frac{R}{Z(R)} \cong \mathbb{Z}_{p} \times \mathbb{Z}_{p}$ then $\Gamma_{R}$ is neither super hyperenergetic nor super borderenergetic.

Proof. We have $\left|v\left(\Gamma_{R}\right)\right|=|Z(R)|\left(p^{2}-1\right)$, since $|R|=p^{2}|Z(R)|$ and $\left|v\left(\Gamma_{R}\right)\right|=|R|-|Z(R)|$. Therefore

$$
E\left(K_{\left|v\left(\Gamma_{R}\right)\right|}\right)=L E\left(K_{\left|v\left(\Gamma_{R}\right)\right|}\right)=L E^{+}\left(K_{\left|v\left(\Gamma_{R}\right)\right|}\right)=2\left(|Z(R)|\left(p^{2}-1\right)-1\right) .
$$

Since $2\left(|Z(R)|\left(p^{2}-1\right)\right)-(p+1)<2\left(|Z(R)|\left(p^{2}-1\right)-1\right)$, by (3.1) the result follows.

Corollary 4.2. $\Gamma_{R}$ is neither super hyperenergetic nor super borderenergetic if

(a) $R$ is of order $p^{2}$.

(b) $R$ is of order $p^{3}$ with unity.

(c) $R$ is a 4-centralizer ring. 
(d) $R$ is a 5-centralizer ring.

(e) $R$ is a $(p+2)$-centralizer p-ring.

(f) $p$ is the smallest prime dividing $|R|$ and $\operatorname{Pr}(R)=\frac{p^{2}+p-1}{p^{3}}$.

(g) $\operatorname{Pr}(R)=\frac{5}{8}$.

Proof. In any of the above cases, the additive quotient group $\frac{R}{Z(R)}$ is isomorphic to $\mathbb{Z}_{p} \times \mathbb{Z}_{p}$ for some prime $p$. Hence, the result follows from Theorem 4.1.

Theorem 4.3. If $R$ is a non-commutative ring with unity of order $p^{4}$ then $\Gamma_{R}$ is neither super hyperenergetic nor super borderenergetic.

Proof. If $|Z(G)|=p$ then $\left|v\left(\Gamma_{R}\right)\right|=p^{4}-p$. Therefore

$$
\begin{aligned}
E\left(K_{\left|v\left(\Gamma_{R}\right)\right|}\right)=L E\left(K_{\left|v\left(\Gamma_{R}\right)\right|}\right)=L E^{+}\left(K_{\left|v\left(\Gamma_{R}\right)\right|}\right) & =2\left(p^{4}-p-1\right) \\
& =2\left(\left(p^{2}+p+1\right)\left(p^{2}-p\right)-1\right) .
\end{aligned}
$$

We have $2\left(p^{2}+p+1\right)\left(p^{2}-p-1\right)<2\left(p^{4}-p-1\right)$. Also

$$
2 l_{1}\left(p^{2}-p-1\right)+2 l_{2}\left(p^{3}-p-1\right)<2\left(\left(p^{2}+p+1\right)\left(p^{2}-p\right)-1\right)
$$

if $l_{1}, l_{2}$ are positive integers such that $l_{1}+l_{2}(p+1)=p^{2}+p+1$. Hence, the result follows Theorem 3.9 .

If $|Z(G)|=p^{2}$ then $\left|v\left(\Gamma_{R}\right)\right|=p^{4}-p^{2}$. Therefore

$$
E\left(K_{\left|v\left(\Gamma_{R}\right)\right|}\right)=L E\left(K_{\left|v\left(\Gamma_{R}\right)\right|}\right)=L E^{+}\left(K_{\left|v\left(\Gamma_{R}\right)\right|}\right)=2\left(p^{4}-p^{2}-1\right) .
$$

We have

$$
2(p+1)\left(p^{3}-p^{2}-1\right)<2\left(p^{4}-p^{2}-1\right) .
$$

Hence, the result follows Theorem 3.9.

Theorem 4.4. If $R$ is a non-commutative ring with unity of order $p^{5}$ such that $Z(R)$ is not a field then $\Gamma_{R}$ is neither super hyperenergetic nor super borderenergetic.

Proof. If $|Z(R)|=p^{2}$ then $\left|v\left(\Gamma_{R}\right)\right|=p^{5}-p^{2}$. Therefore

$$
\begin{aligned}
E\left(K_{\left|v\left(\Gamma_{R}\right)\right|}\right)=L E\left(K_{\left|v\left(\Gamma_{R}\right)\right|}\right)=L E^{+}\left(K_{\left|v\left(\Gamma_{R}\right)\right|}\right) & =2\left(p^{5}-p^{2}-1\right) \\
& =2\left(\left(p^{3}-p^{2}\right)\left(p^{2}+p+1\right)-1\right) .
\end{aligned}
$$

We have $2\left(p^{2}+p+1\right)\left(p^{3}-p^{2}-1\right)<2\left(p^{5}-p^{2}-1\right)$. Also

$$
2 l_{1}\left(p^{3}-p^{2}-1\right)+2 l_{2}\left(p^{3}-p-1\right)<2\left(\left(p^{3}-p^{2}\right)\left(p^{2}+p+1\right)-1\right)
$$

if $l_{1}, l_{2}$ are positive integers such that $l_{1}+l_{2}(p+1)=p^{2}+p+1$. Hence, the result follows from Theorem 3.10.

If $|Z(R)|=p^{3}$ then $\left|v\left(\Gamma_{R}\right)\right|=p^{5}-p^{3}$. Therefore

$$
E\left(K_{\left|v\left(\Gamma_{R}\right)\right|}\right)=L E\left(K_{\left|v\left(\Gamma_{R}\right)\right|}\right)=L E^{+}\left(K_{\left|v\left(\Gamma_{R}\right)\right|}\right)=2\left(p^{5}-p^{3}-1\right) .
$$

We have $2(p+1)\left(p^{4}-p^{3}-1\right)<2\left(p^{5}-p^{3}-1\right)$. Hence, the result follows from Theorem 3.10 .

Theorem 4.5. Let $R$ be a non-commutative ring of order pq such that $Z(R)=\{0\}$. Then $\Gamma_{R}$ is neither super hyperenergetic nor super borderenergetic.

Proof. We have $\left|v\left(\Gamma_{R}\right)\right|=p q-1$. Therefore

$$
E\left(K_{\left|v\left(\Gamma_{R}\right)\right|}\right)=L E\left(K_{\left|v\left(\Gamma_{R}\right)\right|}\right)=L E^{+}\left(K_{\left|v\left(\Gamma_{R}\right)\right|}\right)=2(p q-2) .
$$

If $t \in\{p, q\}$ and $(t-1) \mid(p q-1)$ then $\frac{2(p q-1)(t-2)}{t-1}<2(p q-2)$. Also $2 l_{1}(p-2)+2 l_{2}(q-2)<$ $2(p q-2)$ if $l_{1}, l_{2}$ are positive integers and $l_{1}(p-1)+l_{2}(q-1)=p q-1$. Hence, the result follows from Theorem 3.11. 
Theorem 4.6. Let $R$ be a non-commutative ring of order $p^{2} q$ such that $Z(R)=\{0\}$. Then $\Gamma_{R}$ is neither super hyperenergetic nor super borderenergetic.

Proof. We have $\left|v\left(\Gamma_{R}\right)\right|=p^{2} q-1$. Therefore

$$
E\left(K_{\left|v\left(\Gamma_{R}\right)\right|}\right)=L E\left(K_{\left|v\left(\Gamma_{R}\right)\right|}\right)=L E^{+}\left(K_{\left|v\left(\Gamma_{R}\right)\right|}\right)=2\left(p^{2} q-2\right) .
$$

If $t \in\left\{p, q, p^{2}, p q\right\}$ and $(t-1) \mid\left(p^{2} q-1\right)$ then we have $\frac{2\left(p^{2} q-1\right)(t-2)}{t-1}<2\left(p^{2} q-2\right)$. Also, $2\left(p^{2} q-1-\left(l_{1}+l_{2}+l_{3}+l_{4}\right)<2\left(p^{2} q-2\right)\right.$ if $l_{1}, l_{2}$ are positive integers such that $l_{1}(p-$ 1) $+l_{2}(q-1)+l_{3}\left(p^{2}-1\right)+l_{4}(p q-1)=p^{2} q-1$. Hence, the result follows from Theorem 3.12 .

Theorem 4.7. Let $R$ be a non-commutative ring with unity having order $p^{3} q$. If $|Z(R)|$ is not a prime then $\Gamma_{R}$ is neither super hyperenergetic nor super borderenergetic.

Proof. If $|Z(R)|=p q$ then $\left|v\left(\Gamma_{R}\right)\right|=p^{3} q-p q$. Therefore

$$
E\left(K_{\left|v\left(\Gamma_{R}\right)\right|}\right)=L E\left(K_{\left|v\left(\Gamma_{R}\right)\right|}\right)=L E^{+}\left(K_{\left|v\left(\Gamma_{R}\right)\right|}\right)=2\left(p^{3} q-p q-1\right) .
$$

We have $2(p+1)\left(p^{2} q-p q-1\right)<2\left(p^{3} q-p q-1\right)$. Hence, the result follows from Theorem 3.13 .

$$
\begin{aligned}
& \text { If }|Z(R)|=p^{2} \text { then }\left|v\left(\Gamma_{R}\right)\right|=p^{3} q-p^{2} \text {. Therefore } \\
& \qquad E\left(K_{\left|v\left(\Gamma_{R}\right)\right|}\right)=L E\left(K_{\left|v\left(\Gamma_{R}\right)\right|}\right)=L E^{+}\left(K_{\left|v\left(\Gamma_{R}\right)\right|}\right)=2\left(p^{3} q-p^{2}-1\right) .
\end{aligned}
$$

We have $\frac{2(p q-1)\left(p^{3}-p^{2}-1\right)}{p-1}<2\left(p^{3} q-p^{2}-1\right), \frac{2(p q-1)\left(p^{2} q-p^{2}-1\right)}{q-1}<2\left(p^{3} q-p^{2}-1\right)$ and $2 l_{1}\left(p^{3}-\right.$ $\left.p^{2}-1\right)+2 l_{2}\left(p^{2} q-p^{2}-1\right)<2\left(p^{3} q-p^{2}-1\right)$ if $(p-1)|(p q-1),(q-1)|(p q-1)$ and $l_{1}(p-1)+l_{2}(q-1)=p q-1$ respectively. Hence, the result follows from Theorem 3.14.

We conclude this paper with the following general result.

Theorem 4.8. If $R$ is finite CC-ring then $\Gamma_{R}$ is neither super hyperenergetic nor super borderenergetic.

Proof. We have $\left|v\left(\Gamma_{R}\right)\right|=|R|-|Z(R)|$. Therefore

$$
E\left(K_{\left|v\left(\Gamma_{R}\right)\right|}\right)=L E\left(K_{\left|v\left(\Gamma_{R}\right)\right|}\right)=L E^{+}\left(K_{\left|v\left(\Gamma_{R}\right)\right|}\right)=2(|R|-|Z(R)|-1) .
$$

Also, $2(|R|-|Z(R)|-n)<2(|R|-|Z(R)|-1)$, where $n$ is the number of distinct centralizers of non-central elements of $R$. Hence, the results follows from Theorem 3.15.

Acknowledgment. The authors would like to thank the referee for his/her valuable comments and suggestions. The first author is thankful to Indian Council for Cultural Relations for the ICCR Scholarship.

\section{References}

[1] A. Abdollahi, Commuting graphs of full matrix rings over finite fields, Linear Algebra Appl. 428, 2947-2954, 2008.

[2] M. Afkhami, Z. Barati, N. Hoseini and K. Khashyarmanesh, A generalization of commuting graphs, Discrete Math. Algorithm. Appl. 7 (1), 1450068 (11 pages), 2015.

[3] S. Akbari, M. Ghandehari, M. Hadian and A. Mohammadian, On commuting graphs of semisimple rings, Linear Algebra Appl. 390, 345-355, 2004.

[4] S.M. Buckley, D. Machale, and A.N. Shé, Finite rings with many commuting pairs of elements, available at http://archive.maths.nuim.ie/staff/sbuckley/Papers/ bms.pdf.

[5] J. Dutta and R.K. Nath, Rings having four distinct centralizers, Matrix, M. R. Publications, Assam, 2017, pp. 12-18, Ed. P. Begum. 
[6] P. Dutta and R.K. Nath, A generalization of commuting probability of finite rings, Asian-European J. Math. 11 (2), 1850023 (15 pages), 2018.

[7] P. Dutta and R.K. Nath, On relative commuting probability of finite rings, Miskolc Math. Notes 20 (1), 225-232, 2019.

[8] J. Dutta, D.K. Basnet and R.K. Nath, On commuting probability of finite rings, Indag. Math. 28 (2), 272-282, 2017.

[9] J. Dutta, D.K. Basnet and R.K. Nath, On generalized non-commuting graph of a finite ring, Algebra Colloq. 25 (1), 149-160, 2018.

[10] J. Dutta, D.K. Basnet and R.K. Nath, A note on n-centralizer finite rings, An. Stiint. Univ. Al. I. Cuza Iasi Math. LXIV (f.1), 161-171, 2018.

[11] J. Dutta, D.K. Basnet and R.K. Nath, Characterizing some rings of finite order, submitted for publication, available at https://arxiv.org/pdf/1510.08207.pdf.

[12] J. Dutta, W.N.T. Fasfous and R.K. Nath, Spectrum and genus of commuting graphs of some classes of finite rings, Acta Comment. Univ. Tartu. Math. 23 (1), 5-12, 2019.

[13] A. Erfanian, K. Khashyarmanesh and Kh. Nafar, Non-commuting graphs of rings, Discrete Math. Algorithm. Appl. 7 (3), 1550027 (7 pages), 2015.

[14] S.C. Gong, X. Li, G.H. Xu, I. Gutman and B. Furtula, Borderenergetic graphs, MATCH Commun. Math. Comput. Chem. 74, 321-332, 2015.

[15] I. Gutman, Hyperenergetic molecular graphs, J. Serb. Chem. Soc. 64, 199-205, 1999.

[16] D. MacHale, Commutativity in finite rings, Amer. Math. Monthly, 83, 30-32, 1976.

[17] A. Mohammadian, On commuting graphs of finite matrix rings, Comm. Algebra 38, 988-994, 2010.

[18] R.K. Nath, Various spectra of commuting graphs of n-centralizer finite groups, J. Eng. Science and Tech. 10 (2S), 170-172, 2018.

[19] R.K. Nath, A note on super integral rings, Bol. Soc. Paran. Mat. 38 (4), 213-218, 2020.

[20] G.R. Omidi and E. Vatandoost, On the commuting graph of rings, J. Algebra Appl. 10 (3), 521-527, 2011.

[21] F. Tura, L-borderenergetic graphs, MATCH Commun. Math. Comput. Chem. 77, 37-44, 2017.

[22] E. Vatandoost and F. Ramezani, On the commuting graph of some non-commutative rings with unity, J. Linear Topological Algebra, 5 (4), 289-294, 2016.

[23] E. Vatandoost, F. Ramezani and A. Bahraini, On the commuting graph of noncommutative rings of order $p^{n} q$, J. Linear Topological Algebra, 3 (1), 1-6, 2014.

[24] H.B. Walikar, H.S. Ramane and P.R. Hampiholi, On the energy of a graph, Graph Connections, Eds. R. Balakrishnan, H.M. Mulder, A. Vijayakumar., pp. 120-123, Allied Publishers, New Delhi, 1999. 